\title{
Sympathoinhibitory pathway from caudal midline medulla to RVLM is independent of baroreceptor reflex pathway
}

\author{
J. R. Potas and R. A. L. Dampney \\ Department of Physiology and Institute for Biomedical Research, \\ University of Sydney, New South Wales 2006, Australia \\ Submitted 11 September 2002; accepted in final form 28 November 2002
}

\begin{abstract}
Potas, J. R., and R. A. L. Dampney. Sympathoinhibitory pathway from caudal midline medulla to RVLM is independent of baroreceptor reflex pathway. Am J Physiol Regul Integr Comp Physiol 284: R1071-R1078, 2003. First published December 19, 2002; 10.1152/ajpregu.00559.2002._Glutamate stimulation of the caudal midline medulla (CMM) causes profound sympathoinhibition due to GABAergic inhibition of presympathetic neurons in the rostral ventrolateral medulla (RVLM). We investigated whether the sympathoinhibitory pathway from CMM to RVLM, like the central baroreceptor reflex pathway, includes a glutamatergic synapse in the caudal ventrolateral medulla (CVLM). In pentobarbital sodium-anesthetized rats, the RVLM on one side was inhibited by a muscimol microinjection. Then the response evoked by glutamate microinjections into the CMM or by baroreceptor stimulation was determined before and after 1) microinjection of the GABA receptor antagonist bicuculline into the RVLM on the other side or 2) microinjections of the glutamate receptor antagonist kynurenate bilaterally into the CVLM. Bicuculline in the RVLM greatly reduced both CMM- and baroreceptorevoked sympathoinhibition. Compared with the effect of vehicle solution, kynurenate in the CVLM greatly reduced baroreceptor-evoked sympathoinhibition, whereas its effect on CMMevoked sympathoinhibition was not different from that of the vehicle solution. These findings indicate that the output pathway from CMM sympathoinhibitory neurons, unlike the baroreceptor and other reflex sympathoinhibitory pathways, does not include a glutamatergic synapse in the CVLM.
\end{abstract}

glutamatergic neurotransmission; caudal ventrolateral medulla; sympathoexcitatory neurons; central cardiovascular pathways

MICROINJECTION OF NEUROEXCITATORY amino acids into a discrete region within the caudal midline medulla (CMM) of the rabbit or rat causes a profound fall in arterial blood pressure, heart rate (HR), and sympathetic activity $(3,10,28)$. In both species this region is located in the ventral part of the CMM, at the rostrocaudal level corresponding to the middle third of the inferior olive $(3,10,28)$. In the rat, the $\mathrm{CMM}$ depressor region extends rostrocaudally for $\sim 1.5 \mathrm{~mm}(10,28)$ and is centered on the level corresponding to the level 12.8 $\mathrm{mm}$ posterior to bregma, according to the atlas of Paxinos and Watson (20).

Inhibition of neurons or blockade of synaptic transmission within the CMM depressor region does not

Address for reprint requests and other correspondence: R.A.L. Dampney, Dept. of Physiology, F13, The Univ. of Sydney, NSW 2006, Australia (E-mail: rogerd@physiol.usyd.edu.au). alter resting arterial pressure or sympathetic activity $(3,11)$, nor does it affect the cardiovascular reflex effects evoked by stimulation of arterial baroreceptors and cardiopulmonary receptors (11). Thus the CMM depressor region does not appear to have a critical role in regulating the tonic level of arterial pressure or sympathetic activity. On the other hand, there is evidence that this CMM region may mediate depressor and sympathoinhibitory responses arising from supramedullary regions in the brain. For example, there is anatomic and electrophysiological evidence that neurons in the CMM receive descending inputs from the depressor region in the ventrolateral part of the midbrain periaqueductal gray $(10,23,29)$, and it has been suggested that the acute sympathoinhibition that is evoked in severe hemorrhage or as part of other behavioral responses is mediated, at least in part, via this pathway $(7,11)$.

The output pathway via which stimulation of CMM neurons leads to profound sympathoinhibition has not been determined, although it has been shown in the rabbit that it includes a GABAergic synapse in the rostral ventrolateral medulla (RVLM) as a critical component (4). Consistent with this, Verberne et al. (28) showed that electrical or glutamate stimulation of the CMM in the rat inhibited the ongoing activity of RVLM presympathetic neurons. It is well known that RVLM presympathetic neurons are also inhibited via GABAergic synapses in response to stimulation of a variety of peripheral receptors, including arterial baroreceptors, cardiopulmonary receptors, and other vagally innervated receptors $(13,24,25)$. The central pathway mediating the reflex sympathoinhibition evoked by excitation of these receptors also includes a glutamatergic synapse in the CVLM $(9,13,27)$. Furthermore, it has recently been shown that the depressor response evoked by stimulation of afferent fibers within the greater splanchnic nerve is also mediated via a glutamatergic synapse in the CVLM (21).

These previous observations therefore raise the possibility that the inhibitory pathway from the CMM to the RVLM presympathetic neurons includes, like the central pathways subserving sympathoinhibition arising from arterial baroreceptors and a wide range of

The costs of publication of this article were defrayed in part by the payment of page charges. The article must therefore be hereby marked "advertisement" in accordance with 18 U.S.C. Section 1734 solely to indicate this fact. 
other visceral receptors, a glutamatergic synapse in the CVLM. The primary aim of this study was to test this hypothesis. In addition, as a first step, we confirmed that in the rat, as in the rabbit (4), GABAergic transmission in the RVLM is crucial for CMM-evoked sympathoinhibition.

\section{METHODS}

General procedures. All experiments and procedures were performed on male Sprague-Dawley rats and complied with the guidelines of the National Health and Medical Research Council of Australia and of the American Physiological Society. Each animal was anesthetized initially by exposure to a gas mixture of 5\% halothane (Fluothane; Zeneca, Macclesfield, UK) and 30\% oxygen in a container, after which halothane anesthesia was continued via a mask $(1.5-3 \%$ in $30 \%$ oxygen) while cannulations were performed. The right femoral artery and vein were cannulated for the measurement of arterial pressure and the administration of drugs, respectively. The trachea was cannulated and all animals were then artificially ventilated with $1.5 \%$ halothane in $30 \%$ oxygen. The rat was then placed in a stereotaxic frame with the nose positioned at $3.0 \mathrm{~mm}$ below the interaural axis. The halothane ventilation was then replaced by intravenous anesthesia with pentobarbital sodium (Nembutal; Boehringer Ingelheim; bolus injection initially of $45 \mathrm{mg} / \mathrm{kg}$ followed by continuous infusion at the rate of $15-20 \mathrm{mg} \cdot \mathrm{kg}^{-1} \cdot \mathrm{h}^{-1}$ ). Inspiratory oxygen levels were maintained in the range 39 $41 \%$, and expired carbon dioxide levels were maintained in the range $3.5-4.5 \%$. The remaining surgery (exposure of the medulla and renal nerve) was then performed under pentobarbital sodium infusion. The adequacy of the level of anesthesia was ensured by the lack of withdrawal reflexes to toe pinch. Core temperature was maintained at $36.5-37.2^{\circ} \mathrm{C}$ with a servocontrolled heating pad. Mean arterial pressure (MAP) and HR signals were derived from the arterial pressure signal by means of MacLab software (Chart v 4.0).

Renal sympathetic nerve recording. Renal sympathetic nerve activity (RSNA) was recorded from the left renal nerve. After exposure and isolation, the renal nerve was placed on bipolar recording electrodes and covered with mineral oil. The signal was passed through a band-pass filter (20-750 $\mathrm{Hz}$ ) and displayed and monitored on a cathode ray oscilloscope and audio amplifier. The filtered nerve signal was recorded on a MacLab recording system, rectified, and integrated (resetting every $2.5 \mathrm{~s}$ ). At the end of each experiment, lignocaine was administered to the proximal end of the renal nerve, and the remaining signal was taken as the background noise level and was thus subtracted from the level of RSNA recorded during the experiment.

Stimulation of baroreceptors. Arterial baroreceptors were stimulated by raising arterial pressure with an intravenous injection of phenylephrine (5-10 $\mu \mathrm{g}$ in $0.05-0.10 \mathrm{ml}$ saline). The dose of phenylephrine was chosen so as to produce an increase in MAP of 50-70 $\mathrm{mmHg}$, and the gain of the sympathoinhibitory component of the reflex was calculated as the change in RSNA (measured as a percentage of the prestimulus baseline level) divided by the increase in MAP (in $\mathrm{mmHg}$ ).

Intramedullary microinjections. Microinjections into the caudal medulla were made using a glass micropipette held with a micromanipulator. Injections were made by pressure, and the volume was measured by observing the movement of the meniscus in the micropipette using a dissecting microscope. Rostrocaudal, mediolateral, and dorsoventral coordinates of the tip of the micropipette were referred to the calamus scriptorius. Microinjections of glutamate $(50 \mathrm{mM}, 50$ nl) were made into the CMM in the midline, $1.5 \mathrm{~mm}$ rostral and $1.7 \mathrm{~mm}$ ventral to calamus scriptorius. These coordinates were selected because preliminary experiments established that glutamate microinjections at these coordinates consistently evoked a significant depressor response (i.e., decrease in MAP $>20 \mathrm{mmHg}$ ). In the rare cases where a glutamate microinjection into this CMM site failed to evoke a significant depressor and sympathoinhibitory response, the experiment was discontinued. Bilateral microinjections of kynurenate $(27 \mathrm{mM}, 150 \mathrm{nl})$ were placed into either caudal or rostral subregions of the CVLM (CVLMc and CVLMr, respectively). Microinjections into CVLMc were placed $0.5 \mathrm{~mm}$ rostral, $1.8 \mathrm{~mm}$ lateral, and $2.3 \mathrm{~mm}$ ventral, and microinjections into CVLMr were placed at $1.5 \mathrm{~mm}$ rostral, $1.8 \mathrm{~mm}$ lateral, and $2.1 \mathrm{~mm}$ ventral to calamus scriptorius. Again, preliminary experiments established that glutamate microinjections at these coordinates consistently evoked a significant depressor response (i.e., decrease in MAP > $30 \mathrm{mmHg}$ ).

Microinjections of muscimol (10 mM, $150 \mathrm{nl})$ and/or bicuculline $(2 \mathrm{mM}, 150 \mathrm{nl})$ were made into the pressor region in the RVLM, which was identified as the site at which a microinjection of glutamate $(50 \mathrm{mM}, 50 \mathrm{nl})$ evoked a pressor response of at least $20 \mathrm{mmHg}$. Usually, less than three penetrations were required to identify the RVLM pressor region on each side. The coordinates of the RVLM pressor region were usually at $2.3 \mathrm{~mm}$ rostral, $1.8 \mathrm{~mm}$ lateral, and $2.1 \mathrm{~mm}$ ventral to calamus scriptorius. In most experiments, fluorescent-labeled microspheres were added to the injectate to enable histological examination of injection sites.

Experimental procedure. The basic experimental strategy was to test the effect of blockade of GABAergic neurotransmission in the RVLM, or of glutamate neurotransmission in the CVLM, on the sympathoinhibitory response evoked from the CMM as well as the reflex sympathoinhibitory response evoked by baroreceptor stimulation. However, bilateral blockade of GABA receptors in the RVLM, or of glutamate receptors in the CVLM, results in large increases in baseline MAP and RSNA $(4,17,18)$. Therefore, to avoid this problem, a microinjection of muscimol, a long-lasting GABA receptor agonist (8), was first made into the RVLM on the left side in all experiments. Subsequently, in the first series of experiments, the effects on RSNA of stimulation of the CMM (by glutamate microinjection) and of stimulation of arterial baroreceptors (by raising MAP with phenylephrine injection) were tested before and after unilateral injection of the GABA receptor antagonist bicuculline into the RVLM on the other (right) side. In the second series of experiments, the effects on RSNA of stimulation of the CMM and of stimulation of arterial baroreceptors were tested before and after bilateral injections of kynurenate into the CVLM.

The time frame for the sequence of microinjections was as follows. After microinjection of muscimol into the left RVLM, there was a waiting period of $7-15$ min after which a control microinjection of glutamate was made into the CMM. After the CMM-evoked response, when the cardiovascular variables had stabilized, there was a further waiting period of $\sim 2$ min, after which the baroreceptor reflex response was evoked. There was then a further waiting period of 10-15 min, after which bicuculline was injected into the right RVLM or kynurenate was injected bilaterally into the CVLM. When the cardiovascular variables had stabilized after these injections, there was a further waiting period of $\sim 5 \mathrm{~min}$, after which a microinjection of glutamate was again made into the CMM. Finally, after the CMM-evoked response, there was a further waiting period of $\sim 2$ min, after which the baroreceptor reflex response was again evoked. In control experiments, the procedures were the same, except that the vehicle solu- 
Table 1. Effects on cardiovascular variables of CMM and baroreceptor stimulation under normal conditions and following unilateral inactivation of the RVLM

\begin{tabular}{|c|c|c|}
\hline & $\begin{array}{c}\text { Normal } \\
\text { Conditions } \\
(n=5)\end{array}$ & $\begin{array}{c}\text { Unilateral RVLM } \\
\text { Inactivation } \\
(n=32)\end{array}$ \\
\hline \multicolumn{3}{|c|}{ Glutamate injection into CMM } \\
\hline$\Delta \mathrm{MAP}, \mathrm{mmHg}$ & $-39 \pm 5$ & $-9 \pm 1^{*}$ \\
\hline$\Delta \mathrm{HR}$, beats/min & $-33 \pm 13$ & $-9 \pm 2 *$ \\
\hline$\triangle$ RSNA, \%baseline & $-59 \pm 7$ & $-52 \pm 4$ \\
\hline \multicolumn{3}{|l|}{ Phenylephrine infusion } \\
\hline$\Delta \mathrm{MAP}, \mathrm{mmHg}$ & $64 \pm 2$ & $67 \pm 3$ \\
\hline$\Delta \mathrm{HR}$, beats $/ \mathrm{min}$ & $-45 \pm 10$ & $-29 \pm 3$ \\
\hline$\triangle$ RSNA, \%baseline & $-76 \pm 4$ & $-58 \pm 4$ \\
\hline
\end{tabular}

Values are mean \pm SE. MAP, mean arterial pressure; HR, heart rate; RSNA, renal sympathetic nerve activity. $* P<0.01$ vs. normal conditions.

tion (artificial cerebrospinal fluid, $144 \mathrm{mM} \mathrm{NaCl}, 1.2 \mathrm{mM}$ $\mathrm{CaCl}_{2}, 2.8 \mathrm{mM} \mathrm{KCl}, 0.9 \mathrm{mM} \mathrm{MgCl}{ }_{2}$ ) was microinjected instead of bicuculline into the RVLM (first series of experiments) or instead of kynurenate into the CVLM (second series of experiments).

Finally, the effects of CMM and baroreceptor stimulation on RSNA were also measured in a separate group of animals in which no prior injection of muscimol was first made into the RVLM on one side.

Histology. At the end of the experiment, intracardiac perfusion of $500 \mathrm{ml}$ of $0.9 \%$ saline followed by $500 \mathrm{ml}$ of $0.1 \mathrm{M}$ phosphate buffer of $\mathrm{pH} 7.4$ containing $4 \%$ paraformaldehyde was carried out. Brains were then removed and placed in $30 \%$ sucrose solution for several days at $4^{\circ} \mathrm{C}$, after which $50-\mu \mathrm{m}$-thick sections were cut on a freezing microtome and mounted on glass slides. Two adjacent series were collected at $250-\mu \mathrm{m}$ intervals. Before being placed under coverslips, one series was stained for Nissl bodies. Brain sections were examined under the fluorescence microscope to confirm the location of injections into the medulla.

Data analysis. The baseline values for MAP, HR, and RSNA were measured as the average values for each of these variables over the 12.5 -s period before the stimulus (i.e., CMM stimulation with glutamate or baroreceptor stimulation via the phenylephrine-induced increase in MAP). The change in RSNA evoked by each stimulus was measured at the percentage maximum change in the integrated RSNA (averaged over successive 5-s periods after each stimulus) relative to the prestimulus baseline level. Comparisons of responses evoked by each stimulus in the same animal before and after either blockade of GABAergic neurotransmission in the RVLM or glutamatergic neurotransmission in the CVLM were made using a paired $t$-test. Comparisons of responses in different groups of animals were made using an unpaired $t$-test. A $P$ value of $<0.05$ was taken to indicate a statistically significant difference. All values are expressed as means $\pm \mathrm{SE}$.

\section{RESULTS}

Effects of unilateral injection of muscimol into the RVLM on responses evoked by CMM and baroreceptor stimulation. In one group of five intact rats, the effects of glutamate microinjections into the CMM and of baroreceptor stimulation were tested before any other procedures were performed. The baseline levels of MAP and HR in this group of animals were $104 \pm 6$
$\mathrm{mmHg}$ and $340 \pm 16$ beats/min, respectively. Glutamate microinjections $(2.5 \mathrm{nmol})$ into the CMM in these rats consistently evoked large decreases in MAP, HR, and RSNA (Table 1, Fig. 1). Baroreceptor stimulation (via a phenylephrine-induced increase in MAP) also resulted in a reflex bradycardia, and a profound reflex sympathoinhibition (Table 1).

As explained above in METHODS (see Experimental procedure), in all the other series of experiments the long-lasting GABA receptor agonist muscimol (8) was first injected into the RVLM on one side to prevent the very large increases in baseline MAP that occur after bilateral blockade of GABA receptors in the RVLM or of glutamate receptors in the CVLM. Before unilateral injection of muscimol into the left RVLM, the baseline levels of MAP and HR in all these experiments $(n=32$ in total) were $98 \pm 2 \mathrm{mmHg}$ and $321 \pm 6$ beats $/ \mathrm{min}$. After unilateral muscimol injection, the baseline levels of MAP and HR decreased significantly to $77 \pm 2$ $\mathrm{mmHg}(P<0.01)$ and $310 \pm 5$ beats/min $(P<0.05)$, respectively, and the baseline level of RSNA decreased significantly to $79 \pm 3 \%(P<0.01)$ of the original baseline level. Subsequently, glutamate microinjection into the CMM consistently evoked small decreases in MAP and HR but a large decrease in RSNA (Table 1). The depressor and bradycardic responses evoked by CMM stimulation in rats with unilateral inactivation of the RVLM were significantly less than these responses in the intact group (Table 1). On the other hand, the magnitudes of the renal sympathoinhibitory responses were not significantly different in the two cases (Table 1), although the duration of the sympathoinhibitory response $(33 \pm 3 \mathrm{~s})$ in the group with unilateral inactivation of the RVLM was significantly less than the duration of this response in the intact group (58 $\pm 6 \mathrm{~s}, P<0.01$ ). Although unilateral microinjections of muscimol into the left RVLM reduced the magnitude of the reflex bradycardia and renal sympathoinhibition associated with phenylephrine-induced increases in MAP, these reductions were not significant compared with the intact group (Table 1).

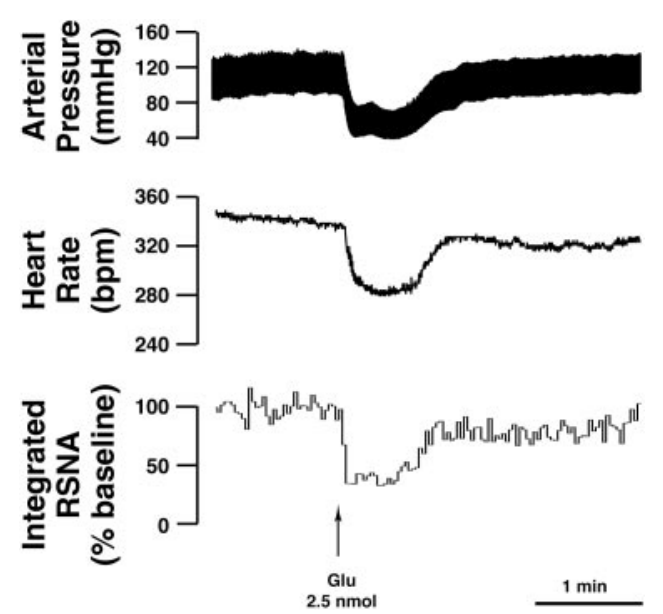

Fig. 1. Example of the cardiovascular response evoked by microinjection of glutamate (Glu; $2.5 \mathrm{nmol})$ into the caudal midline medulla (CMM). RSNA, renal sympathetic nerve activity; bpm, beats/min. 
Effects of microinjection of bicuculline into the right RVLM on CMM- and baroreceptor-evoked sympathoinhibition. Subsequent to unilateral microinjection of muscimol into the left RVLM, microinjection of the vehicle solution into the right RVLM had no significant effect on the baseline levels of MAP, HR, or RSNA (Table 2) nor on the magnitude of the sympathoinhibitory response evoked by glutamate stimulation of the CMM or the gain of the baroreceptor-sympathetic reflex (Fig. 2). After microinjection of bicuculline into the right RVLM, the baseline level of RSNA was greatly increased (Table 2). There was also an increase in the baseline level of MAP, although this just failed to achieve statistical significance $(P=0.059)$. The CMMand baroreceptor-evoked sympathoinhibitory responses were greatly reduced after bicuculline injection (Figs. 2 and 3). When expressed as a percentage of the response before microinjection of bicuculline into the RVLM, the CMM-evoked sympathoinhibitory response and the baroreceptor-sympathetic reflex gain were reduced by $71 \pm 3$ and $72 \pm 12 \%$, respectively.

Effects of microinjections of kynurenate into the CVLM on CMM- and baroreceptor-evoked sympathoinhibition. Subsequent to unilateral microinjection of muscimol in the left RVLM, bilateral microinjection of the vehicle solution into the CVLM had no significant effect on the baseline levels of MAP, HR, and RSNA (Table 2), but did result in a moderate but significant decrease in the magnitudes of both the CMM-evoked and baroreceptor-evoked sympathoinhibitory responses (Fig. 4). When expressed as a percentage of their respective control responses before vehicle injections into the CVLM, these sympathoinhibitory responses were reduced by $33 \pm 12$ and $33 \pm 13 \%$, respectively.

As described in METHODS, bilateral injections of kynurenate were made into either a more rostral or more caudal level of the CVLM at 0.8 and $1.8 \mathrm{~mm}$, respectively, caudal to the RVLM. This was done because sympathoinhibitory neurons in the CVLM appear to be distributed over a considerable rostrocaudal distance (5). Furthermore, there is evidence that baroreceptor interneurons in the CVLM are concen-

Table 2. Baseline levels of MAP, HR, and RSNA before and after injections of various compounds into the RVLM or CVLM

\begin{tabular}{lccccc}
\hline \hline & \multicolumn{2}{c}{ Injections into RVLM } & & \multicolumn{2}{c}{ Injections into CVLM } \\
\cline { 2 - 3 } & $\begin{array}{c}\text { Vehicle } \\
(n=4)\end{array}$ & $\begin{array}{c}\text { Bicuculline } \\
(n=5)\end{array}$ & & $\begin{array}{c}\text { Vehicle } \\
(n=8)\end{array}$ & $\begin{array}{c}\text { Kynurenate } \\
(n=15)\end{array}$ \\
\hline $\begin{array}{l}\text { Before injection } \\
\text { MAP, mmHg }\end{array}$ & $77 \pm 3$ & $72 \pm 4$ & & $78 \pm 3$ & $77 \pm 3$ \\
$\begin{array}{l}\text { HR, beats/min } \\
\text { RSNA, \%original }\end{array}$ & $325 \pm 11$ & $307 \pm 17$ & & $318 \pm 8$ & $302 \pm 6$ \\
$\quad$ baseline level & $76 \pm 5$ & $89 \pm 7$ & & $81 \pm 4$ & $76 \pm 6$ \\
$\begin{array}{c}\text { After injection } \\
\text { MAP, mmHg }\end{array}$ & $79 \pm 4$ & $109 \pm 14$ & $72 \pm 3$ & $82 \pm 5$ \\
$\begin{array}{l}\text { HR, beats/min } \\
\text { RSNA, \%original } \\
\text { baseline level }\end{array}$ & $373 \pm 27$ & $344 \pm 25$ & $298 \pm 19$ & $291 \pm 12$ \\
\hline
\end{tabular}

Values are mean $\pm \mathrm{SE} . * P<0.01$ vs. before injection.
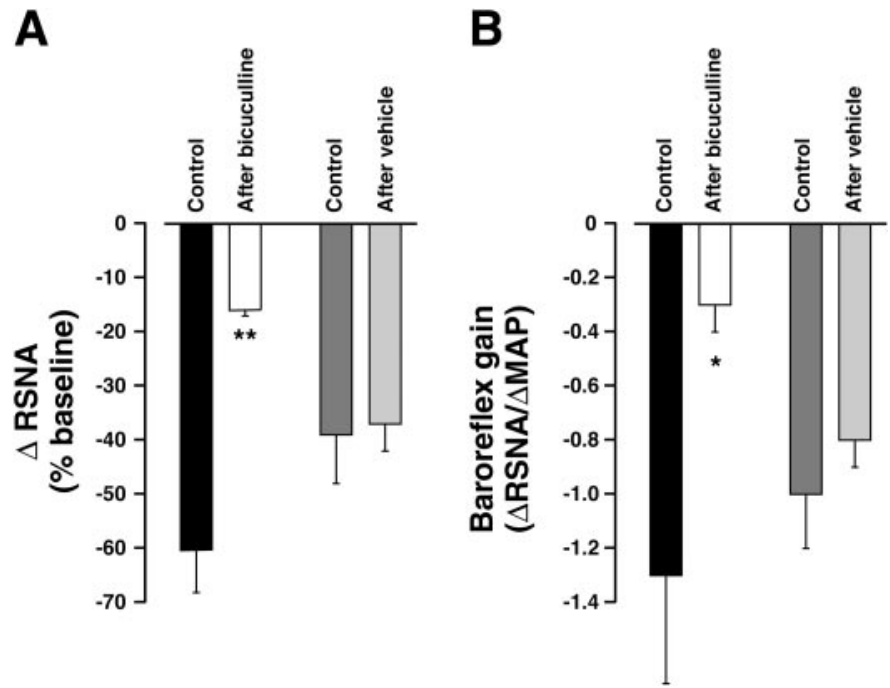

Fig. 2. Grouped results showing effects of GABA-receptor blockade in the right rostral ventrolateral medulla (RVLM) on sympathoinhibition evoked by CMM or baroreceptor stimulation. In all experiments, muscimol (1.5 nmol) was first injected into the left RVLM. The changes in RSNA evoked by microinjection of glutamate (2.5 $\mathrm{nmol}$ ) into the CMM $(A)$ and also the magnitude of the baroreceptorsympathetic reflex gain $(B)$ were then determined before and after microinjection of either bicuculline (300 pmol; $n=5)$ or vehicle solution $(n=4)$ into the right RVLM. $* P<0.05$ and $* * P<0.01$ vs. control response. In each case, the change in RSNA is shown as the percentage change with respect to the baseline level immediately before the CMM or baroreflex response was evoked.

trated in its more rostral portion (5) so that it was thought possible that the effect of kynurenate injections on CMM-evoked sympathoinhibition may differ according to the rostrocaudal level of the injection.

There was, however, no significant difference in the effects of kynurenate injections on the CMM- and baroreceptor-evoked sympathoinhibition with respect to their rostrocaudal location in the CVLM, and so the results from these two groups of experiments were pooled. After bilateral microinjection of kynurenate into the CVLM, there was no significant change in the baseline levels of MAP and HR, but the baseline level of RSNA was significantly increased with respect to the baseline level before kynurenate injections (Table 2), although it was not significantly changed with respect to the original baseline level $(P>0.25)$. The sympathoinhibitory response evoked by glutamate stimulation of the CMM was reduced to a moderate but significant extent, but that evoked by baroreceptor stimulation was reduced to a much greater extent (Figs. 4 and 5). When expressed as a percentage of their respective control responses before kynurenate injections into the CVLM, the CMM-evoked sympathoinhibitory response was reduced by $24 \pm 12 \%$, whereas the baroreceptor-sympathetic reflex gain was reduced by $76 \pm 5 \%$. The moderate reduction in the CMM-evoked sympathoinhibitory response after injections of kynurenate into the CVLM was not significantly different from that which occurred after injections of the vehicle, whereas in the same experiments the baroreceptor-evoked sympathoinhibitory response was reduced to a much greater extent after injec- 
A
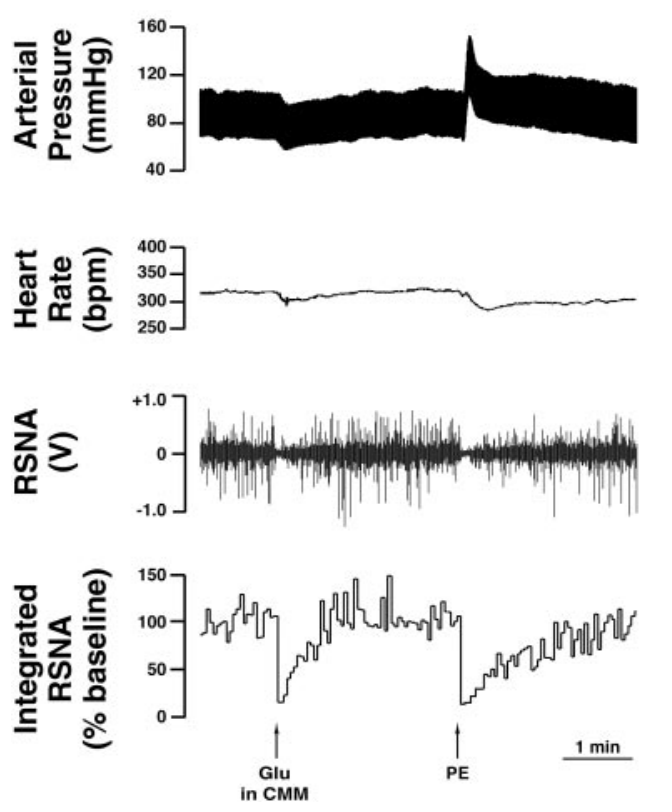

B
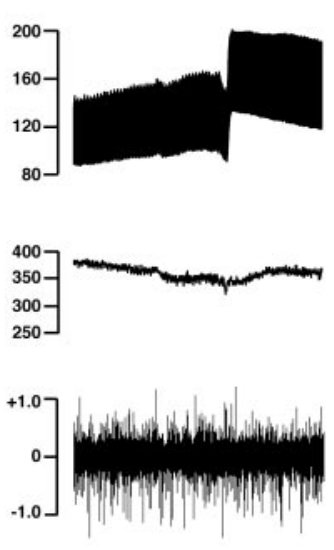

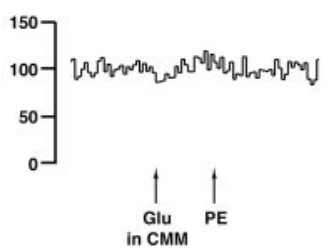

Fig. 3. Example of effects on RSNA evoked by microinjection of Glu ( $2.5 \mathrm{nmol})$ into the CMM and by a phenylephrine (PE)-induced increase in arterial pressure before $(A)$ and after $(B)$ unilateral microinjection of bicuculline (300 pmol) into the RVLM on the right side in one experiment. [A microinjection of muscimol (1.5 $\mathrm{nmol}$ ) had first been made into the RVLM on the left side.] The scale for the raw RSNA signal is the same in both $A$ and $B$. tions of kynurenate compared with the vehicle solution $(P<0.01)$.

Location of injection sites in the CMM, CVLM, and $R V L M$. Histological examination showed that the injection sites in the CMM depressor region were located in the midline at the level corresponding most closely to the level $12.80 \mathrm{~mm}$ posterior to bregma, according to the atlas of Paxinos and Watson (20). In the RVLM, the

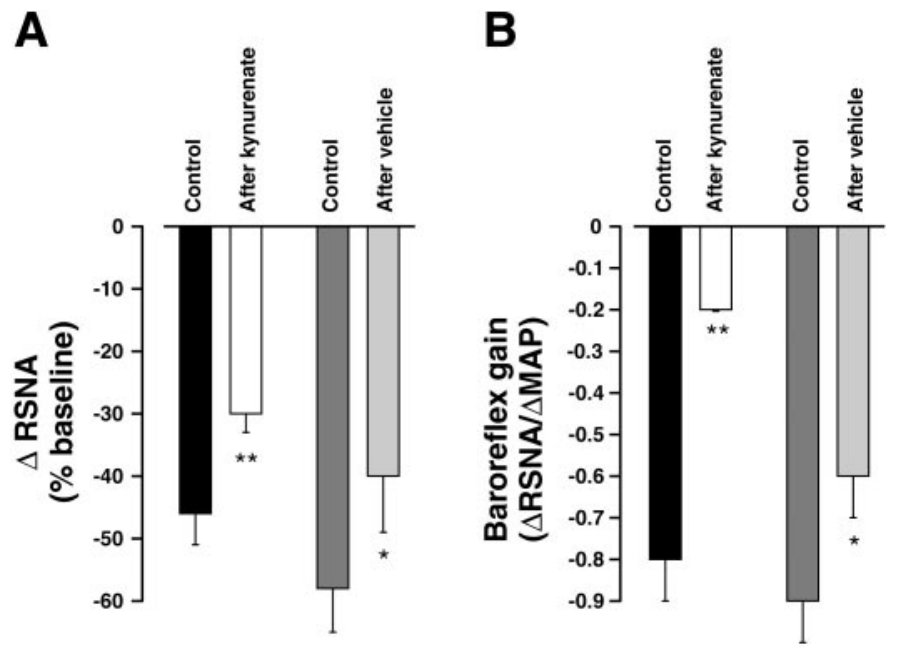

Fig. 4. Grouped results showing effects of Glu-receptor blockade in the caudal ventrolateral medulla (CVLM) on sympathoinhibition evoked by CMM or baroreceptor stimulation. In all experiments, muscimol $(1.5 \mathrm{nmol})$ was first injected into the left RVLM. The changes in RSNA evoked by microinjection of Glu $(2.5 \mathrm{nmol})$ into the CMM $(A)$ and also the magnitude of the baroreceptor-sympathetic reflex gain $(B)$ were then determined before and after bilateral microinjections of either kynurenate $(4 \mathrm{nmol} ; n=15)$ or vehicle solution $(n=8)$ into the CVLM. $* P<0.05$ and $* * P<0.01$ vs. control response. In each case, the change in RSNA is shown as the percentage change with respect to the baseline level immediately before the CMM or baroreflex response was evoked. injection sites were located just ventral to the nucleus ambiguus at the level just caudal to the caudal pole of the facial nucleus [i.e., corresponding most closely to the levels 11.80 or $11.96 \mathrm{~mm}$ posterior to bregma (20)]. Injection sites in the rostral and caudal subregions of CVLM were also located ventral to the nucleus ambiguus, but at levels that corresponded most closely to the levels 12.8 and $13.8 \mathrm{~mm}$ posterior to bregma, respectively (20). Examples of injection sites in the CMM, RVLM, and rostral and caudal subregions of CVLM are shown in Fig. 6, $A$ and $B$.

\section{DISCUSSION}

The results of this study indicate that in the rat, as in the rabbit, the powerful sympathoinhibition that is evoked by stimulation of neurons in the CMM is mediated via GABAergic inhibition of RVLM sympathoexcitatory neurons, as is the case for baroreceptor reflex sympathoinhibition. The main new finding, however, is that unlike the reflex sympathoinhibition arising from baroreceptor stimulation, sympathoinhibition evoked by glutamate stimulation of the CMM did not appear to be affected by blockade of ionotropic glutamate receptors in the CVLM. This finding indicates that, in contrast to the central pathways mediating reflex sympathoinhibition arising from stimulation of baroreceptors and many other peripheral receptors, the sympathoinhibitory pathway from the CMM to the RVLM does not include a glutamatergic synapse within the CVLM. The physiological implications of this finding are discussed below, after some methodological issues are considered.

Methodological considerations. Apart from the first series of experiments in which the effects of glutamate stimulation of the CMM was measured, in all other experiments a microinjection of the long-lasting GABA 
Fig. 5. Example of effects on RSNA evoked by microinjection of Glu $(2.5 \mathrm{nmol})$ into the CMM and by a PE-induced increase in arterial pressure before $(A)$ and after $(B)$ bilateral microinjections of kynurenate $(4 \mathrm{nmol})$ into the CVLM in one experiment. [A microinjection of muscimol $(1.5 \mathrm{nmol})$ had first been made into the RVLM on the left side.]
A

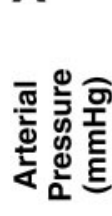

180
$140-$
$100-$

60

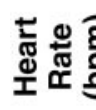

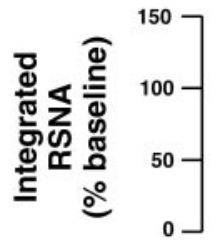
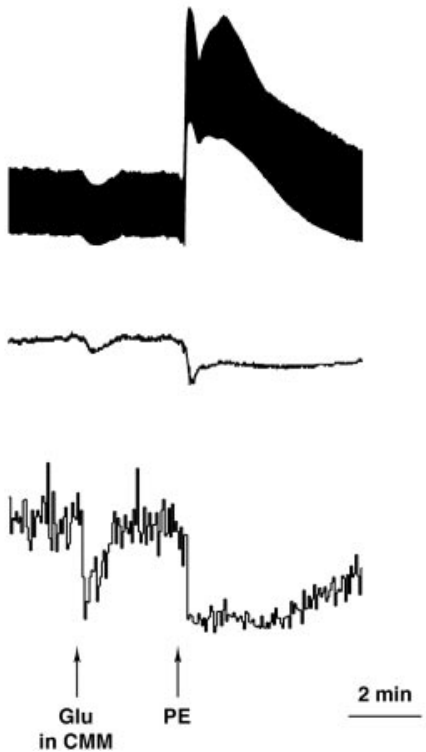

B
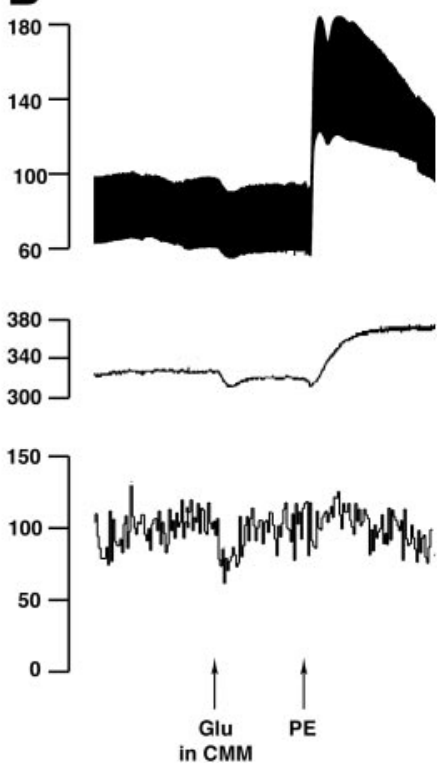

receptor agonist muscimol (8) was first made unilaterally into the RVLM. The purpose of this was to inactivate the RVLM on one side, so that, subsequently, when bicuculline was injected into the RVLM on the other side, or kynurenate was injected into the CVLM on both sides, the baseline arterial pressure and sympathetic activity would not increase to the same extent

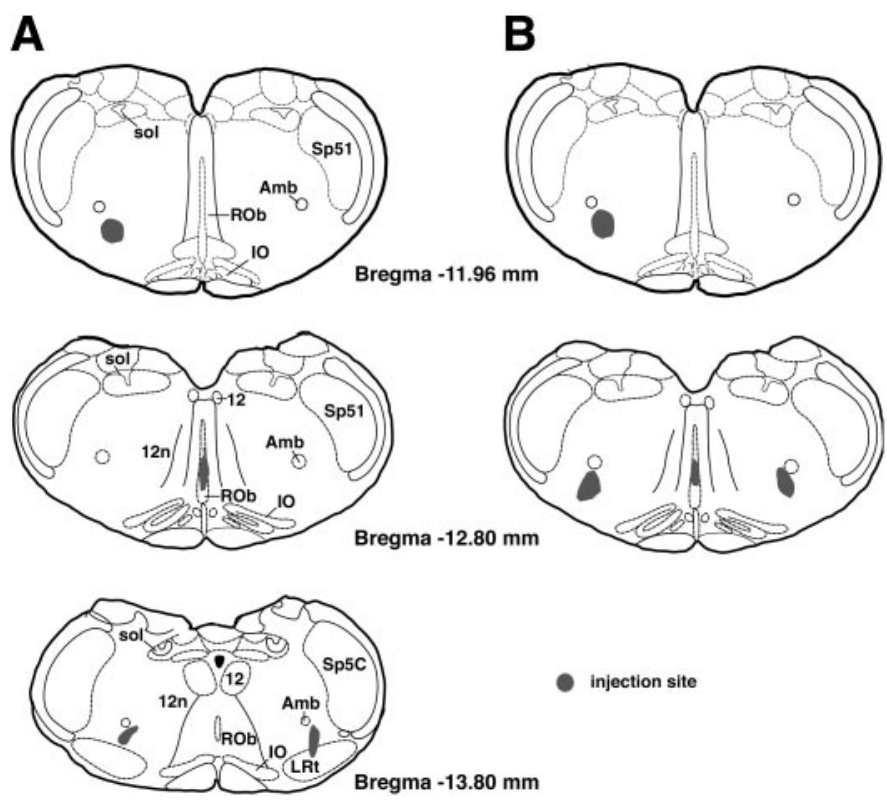

Fig. 6. Injection sites as determined by histological analysis in 2 experiments drawn on to the standard sections of the medulla oblongata from the atlas of Paxinos and Watson (20). In both experiments, bilateral injections of kynurenate were made into the CVLM, at the level of the caudal $(A)$ or rostral $(B)$ parts of CVLM. The injection sites for muscimol into the left RVLM (top) and for Glu into the CMM (middle) are also shown. sol, solitary tract; ROb, raphe obscurus; Amb, nucleus ambiguus; SP51, spinal trigeminal nucleus; IO, inferior olive; 12, hypoglossal nucleus; 12n, hypoglossal nerve; LRt, lateral reticular nucleus; Sp5C, caudal spinal trigeminal nucleus. as is known to occur when RVLM neurons on both sides are disinhibited after either bilateral blockade of GABA receptors in the RVLM or of glutamate receptors in the CVLM $(14,17,18)$. We previously showed that the inhibitory effects of muscimol in the RVLM last for at least $3 \mathrm{~h}$ (12). The inhibitory effects of muscimol in the RVLM, therefore, would have persisted for the entire duration of the experiment, because all experimental procedures were completed within $\sim 1 \mathrm{~h}$ after the injection of muscimol.

Unilateral injection of muscimol into the RVLM did reduce the baseline level of MAP but did not significantly affect the magnitude of the sympathoinhibition evoked by glutamate stimulation of the CMM and resulted in only a small (but not statistically significant) decrease in the magnitude of the baroreceptor reflex sympathoinhibition evoked by a phenylephrine-induced increase in MAP. At the same time, unilateral inhibition of the RVLM did significantly reduce the magnitude of the depressor and bradycardic responses to CMM stimulation. This indicates that unilateral inhibition of the RVLM had nonuniform effects on the degree of CMMevoked inhibition of the sympathetic outflows to the heart and different vascular beds. The reason for this nonuniformity is not clear, but one possibility is that, compared with renal sympathetic preganglionic neurons, the responsiveness of sympathetic preganglionic neurons regulating other vascular beds is much more dependent on bilateral inputs from the RVLM. Further studies are needed to answer this question.

The basic experimental strategy in this study was to compare, in the same experiments, the effects of blockade of GABA receptors in the RVLM or of glutamate receptors in the CVLM on CMM-evoked sympathoinhibition and baroreceptor reflex sympathoinhibition. Each experiment therefore served as its own control, because it is well established that baroreceptor reflex sympathoinhibition is dependent on GABA receptors in the RVLM and 
glutamate receptors in the CVLM $(15,25)$. Furthermore, separate series of control experiments were performed to determine the effect of vehicle injection alone on the magnitude of the evoked responses.

After muscimol microinjection into the RVLM on one side in combination with bicuculline injection into the RVLM on the other side, the baseline level of RSNA was greatly increased (to $245 \%$ of the original baseline level, as measured before any intramedullary microinjections were made). Thus it could be argued that the firing rate of presympathetic neurons in the RVLM on the side where bicuculline was injected may have increased to such an extent that these neurons were not capable of being inhibited by an input originating from the CMM, whether is was mediated by GABA receptors or any other receptor. In a previous study, however, we showed that the profound sympathoinhibition evoked by CMM stimulation is unaffected even under conditions in which disinhibition of RVLM neurons occurs in response to baroreceptor unloading (4). In that study, as in the present study, the change in RSNA evoked by CMM stimulation was measured relative to the baseline level of RSNA immediately before the stimulation was applied. Thus this previous observation indicates that the reduction in the magnitude of the CMMevoked decrease in RSNA that we observed after bicuculline injection into the RVLM is unlikely to be due simply to the increased baseline level of activity of RVLM presympathetic neurons or to the method used to measure the degree of evoked sympathoinhibition. Furthermore, there is direct electrophysiological evidence in the rat that the large majority of RVLM presympathetic neurons are profoundly inhibited by stimulation of the CMM (28). Our findings are therefore entirely consistent with this study (28) and, in addition, indicate that in the rat, as in the rabbit (4), the CMM-evoked inhibition of RVLM presympathetic neurons is likely to be mediated by GABA receptors.

In contrast to the effect of bicuculline injection into the RVLM, bilateral injections of kynurenate into the CVLM had no apparent effect on CMM-evoked sympathoinhibition. Although there was a moderate reduction (of $\sim 25 \%$ ) in the magnitude of the evoked sympathoinhibition after kynurenate microinjections into the CVLM compared with the control response, a reduction of similar magnitude was also observed after bilateral injections of vehicle solution into the CVLM. Thus this modest reduction in the magnitude of the CMM-evoked sympathoinhibition is likely to be due to nonspecific effects of the injection itself, such as mechanical distortion of the tissue, rather than to specific blockade of CVLM glutamate receptors.

In contrast to the CMM-evoked response, when the baroreceptor reflex sympathoinhibitory response was evoked in the same experiments, this was reduced to a much greater extent (by $\sim 75 \%$ ) after microinjection of kynurenate, but not vehicle solution, into the CVLM. The effect of kynurenate injections in reducing the baroreceptor reflex sympathoinhibition is expected, because glutamate receptors in the CVLM are known to be a crucial component of the baroreceptor-sympa- thetic reflex pathway (for review, see Ref. 6). Thus our finding that kynurenate in the CVLM had no apparent effect on the CMM-evoked sympathoinhibition indicates that this pathway is not mediated by glutamate receptors in the CVLM.

As mentioned above, the kynurenate microinjections into the CVLM did not completely block the baroreceptor reflex sympathoinhibition. The volume of kynurenate injected $(150 \mathrm{nl})$ was relatively large, so as to maximize the degree of glutamate receptor blockade in the CVLM. This would explain why kynurenate injections into the rostral and caudal portions of the CVLM had similar effects on baroreceptor reflex sympathoinhibition, even though baroreceptor interneurons are believed to be more concentrated in the rostral part of CVLM (5). Thus, because of the large injection volume, kynurenate injected into the caudal portion of the CVLM may have spread to the more rostral portion of CVLM. Despite this, it is still possible that there was incomplete blockade of glutamate receptors in the CVLM, including some that mediate the baroreceptor reflex. Consistent with this, the increase in baseline RSNA that occurred after kynurenate microinjections into the CVLM was modest. Alternatively, the residual baroreceptor reflex sympathoinhibition could be mediated by other pathways, such as a descending pathway that inhibits sympathetic activity at a spinal level (16). Regardless of this, however, the results of our experiments indicate that the sympathoinhibitory pathway from the CMM to the RVLM is quite separate from the sympathoinhibitory pathway activated by stimulation of arterial baroreceptors. It is also separate from the sympathoinhibitory pathways activated by stimulation of a variety of other visceral receptors (e.g., cardiopulmonary receptors and receptors innervated by the superior laryngeal nerve or greater splanchnic nerve), all of which also include a glutamatergic synapse in the CVLM as well as a GABAergic synapse in the RVLM $(9,13,21,24,25,27)$.

What are the possible central pathways mediating CMM-evoked sympathoinhibition? Anatomic studies using anterograde and retrograde transport have shown that there is a projection from the midline medulla to the RVLM, but this appears to arise mainly from neurons located more rostral than the CMM sympathoinhibitory region $(2,19,22,26,30)$. Thus the available evidence suggests that CMM-evoked sympathoinhibition is unlikely to be mediated by a direct monosynaptic projection to RVLM presympathetic neurons. It therefore seems more likely that the sympathoinhibitory pathway from the CMM is polysynaptic, with direct or indirect connections with other medullary or supramedullary nuclei (such as the nucleus of the solitary tract or the KöllikerFuse nucleus in the pons), which are major sources of afferent inputs to the RVLM (6). Further studies are needed to identify this pathway.

\section{Perspectives}

As shown previously $(3,28)$ and confirmed in this study, glutamate stimulation of the CMM region pro- 
duces profound sympathoinhibition similar to that evoked from the CVLM depressor region. This, therefore, raises the important question as to the physiological function of the CMM sympathoinhibitory neurons. The results of the present study indicate that the sympathoinhibitory pathway from the CMM to the RVLM is quite separate from reflex sympathoinhibitory pathways activated by stimulation of arterial baroreceptors and a variety of other visceral receptors. This implies that the CMM neurons do not play an important role in mediating reflex responses arising from these receptors. Consistent with this, Henderson et al. (11) found that the reflex responses evoked by stimulation of arterial baroreceptors or cardiopulmonary receptors were not affected by inactivation of neurons in the CMM. On the other hand, there is anatomic and electrophysiological evidence that neurons in the CMM receive descending inputs from the ventrolateral part of the midbrain periaqueductal gray $(10,23,29)$, which is believed to evoke sympathoinhibition as part of a more generalized passive behavioral response to various environmental stressors (1). Furthermore, Henderson et al. (11) recently proposed that, whereas the CVLM mediates reflex sympathoinhibitory responses evoked from arterial baroreceptors and other peripheral receptors, the CMM mediates sympathoinhibitory responses that are part of more complex behavioral responses. The finding of the present study that the central pathways mediating CMM-evoked and baroreflex sympathoinhibition are quite separate until their ultimate convergence on presympathetic neurons in the RVLM, is consistent with this hypothesis.

We gratefully acknowledge the assistance of Dr. M. Fontes, Dr. J. Polson, and S. Killinger.

The study was supported by the National Health and Medical Research Council of Australia.

\section{REFERENCES}

1. Bandler R, Keay KA, Floyd N, and Price J. Central circuits mediating patterned autonomic activity during active vs. passive emotional coping. Brain Res Bull 53: 95-104, 2000.

2. Chan SHH, Chan JYH, and Ong BT. Anatomic connections between nucleus reticularis rostroventrolateralis and some medullary cardiovascular sites in the rat. Neurosci Lett 71: 277-282, 1986.

3. Coleman MJ and Dampney RAL. Powerful depressor and sympathoinhibitory effects evoked from neurons in the caudal raphe pallidus and obscurus. Am J Physiol Regul Integr Comp Physiol 268: R1295-R1302, 1995.

4. Coleman MJ and Dampney RAL. Sympathoinhibition evoked from caudal midline medulla is mediated by GABA receptors in the rostral VLM. Am J Physiol Regul Integr Comp Physiol 274: R318-R332, 1998.

5. Cravo SL, Morrison SF, and Reis DJ. Differentiation of two cardiovascular regions within caudal ventrolateral medulla. Am J Physiol Regul Integr Comp Physiol 261: R985-R994, 1991.

6. Dampney RAL. Functional organization of central pathways regulating the cardiovascular system. Physiol Rev 74: 323-364, 1994.

7. Dean C and Bago M. Renal sympathoinhibition mediated by $5-\mathrm{HT}_{1 \mathrm{~A}}$ receptors in the RVLM during severe hemorrhage in rats. Am J Physiol Regul Integr Comp Physiol 282: R122-R130, 2002.

8. DeFeudis FV. Binding studies with muscimol: relation to synaptic $\gamma$-aminobutyrate receptors. Neuroscience 5: 675-688, 1980.
9. Gordon FJ. Aortic baroreceptor reflexes are mediated by NMDA receptors in the caudal ventrolateral medulla. Am J Physiol Regul Integr Comp Physiol 252: R628-R633, 1987.

10. Henderson LA, Keay KA, and Bandler R. The ventrolateral periaqueductal gray projects to caudal brainstem depressor regions: a functional-anatomical and physiological study. Neuroscience 82: 201-221, 1998.

11. Henderson LA, Keay KA, and Bandler R. Caudal midline medulla mediates behaviourally-coupled but not baroreceptormediated vasodepression. Neuroscience 98: 779-792, 2000.

12. Horiuchi J and Dampney RAL. Dependence of sympathetic vasomotor tone on bilateral inputs from the rostral ventrolateral medulla. Neurosci Lett 248: 113-116, 1998.

13. Huangfu D and Guyenet PG. Sympatholytic response to stimulation of superior laryngeal nerve in rats. Am J Physiol Regul Integr Comp Physiol 260: R290-R297, 1991.

14. Ito S and Sved AF. Tonic glutamate-mediated control of rostral ventrolateral medulla and sympathetic vasomotor tone. Am $J$ Physiol Regul Integr Comp Physiol 273: R487-R494, 1997.

15. Koshiya N, Huangfu D, and Guyenet PG. Ventrolateral medulla and sympathetic chemoreflex in the rat. Brain Res 609: 174-184, 1993.

16. Lewis DI and Coote JH. Mediation of baroreceptor inhibition of sympathetic nerve activity via both a brainstem and spinal site in rats. J Physiol 481: 197-205, 1994.

17. Masuda N, Ootsuka Y, and Terui N. Neurons in the caudal ventrolateral medulla mediate the somato-sympathetic inhibitory reflex response via GABA receptors in the rostral ventrolateral medulla. J Auton Nerv Syst 40: 91-98, 1992.

18. Miyawaki T, Suzuki S, Minson J, Arnolda L, Chalmers J, Llewellyn-Smith I, and Pilowsky P. Role of AMPA/kainate receptors in transmission of the sympathetic baroreflex in rat CVLM. Am J Physiol Regul Integr Comp Physiol 272: R800R812, 1997.

19. Nicholas AP and Hancock MB. Evidence for projections from the rostral medullary raphe onto medullary catecholamine neurons in the rat. Neurosci Lett 108: 22-28, 1990.

20. Paxinos G and Watson C. The Rat Brain in Stereotaxic Coordinates (3rd ed.). New York: Academic, 1997.

21. Peng YJ, Wang N, Gong QL, and Li P. Caudal ventrolateral medulla mediates the depressor response elicited by the greater splanchnic nerve afferent stimulation in rats. Neurosci Lett 325: 134-138, 2002.

22. Ross CA, Ruggiero DA, and Reis DJ. Projections from the nucleus tractus solitarii to the rostral ventrolateral medulla. J Comp Neurol 242: 511-534, 1985.

23. Snowball RK, Dampney RAL, and Lumb BM. Responses of neurones in the medullary raphe nuclei to inputs from visceral nociceptors and the ventrolateral periaqueductal grey in the rat. Exp Physiol 82: 485-500, 1997.

24. Sun MK and Guyenet PG. Arterial baroreceptor and vagal inputs to sympathoexcitatory neurons in rat medulla. Am $J$ Physiol Regul Integr Comp Physiol 252: R699-R709, 1987.

25. Sun MK and Guyenet PG. GABA-mediated baroreceptor inhibition of reticulospinal neurons. Am J Physiol Regul Integr Comp Physiol 249: R672-R680, 1985.

26. Van Bockstaele EJ, Pieribone VA, and Aston-Jones G. Diverse afferents converge on the nucleus paragigantocellularis in the rat ventrolateral medulla: retrograde and anterograde tracing studies. J Comp Neurol 290: 561-584, 1989.

27. Verberne AJM, Beart PM, and Louis WJ. Excitatory amino acid receptors in the caudal ventrolateral medulla mediate a vagal cardiopulmonary reflex in the rat. Exp Brain Res 78: 185-192, 1989.

28. Verberne AJM, Sartor DM, and Berke A. Midline medullary depressor responses are mediated by inhibition of RVLM sympathoexcitatory neurons in rats. Am J Physiol Regul Integr Comp Physiol 276: R1054-R1062, 1999.

29. Wang WH and Lovick TA. The inhibitory effect of the ventrolateral periaqueductal grey matter on neurones in the rostral ventrolateral medulla involves a relay in the medullary raphe nuclei. Exp Brain Res 94: 295-300, 1993.

30. Zagon A. Internal connections in the rostral ventromedial medulla of the rat. J Auton Nerv Syst 53: 43-56, 1995. 